\title{
A Review Article of A Citric-Acid-Solution Swallowing Test as a Screening Test for Aspiration at Bedside and for the Early Detection of Swallowing Dysfunction
}

\section{Hope E Baylow}

Department of Speech-Language Pathology, Midwestern University, United States

*Corresponding Author: Hope E Baylow, Assistant Professor, Midwestern University, 19555 N 59"h , Glendale, AZ 85308, United States, Tel: 516-457-0357; E-mail: hbaylo@midwestern.edu

Rec date: Feb 15, 2016; Acc date: Mar 04, 2016; Pub date: Mar 10, 2016

Copyright: ( 2016 Baylow HE. This is an open-access article distributed under the terms of the Creative Commons Attribution License, which permits unrestricted use, distribution, and reproduction in any medium, provided the original author and source are credited.

\section{Commentary}

This study compared using the citric-acid-solution swallowing test (CST) to the modified water swallowing test (MWST) to identify silent aspiration and laryngopharyngeal sensory dysfunction in patients presenting with swallowing disorders. The authors stated that they "developed a screening test" for silent aspiration and dysphagia with laryngopharyngeal sensory dysfunction and were attempting to evaluate the usefulness of CST "in detecting aspiration and in evaluating swallowing dysfunction" [1].

According to the American Speech-Language-Hearing Association [2], a swallowing screening is used to recognize deglutition difficulties resulting in pulmonary aspiration, airway obstruction, or compromised nutrition and /or hydration. A screening outcome may lead to a referral for a swallow function assessment or for a referral for other services. A screening typically involves obtaining baseline information regarding the swallowing status of the patient, acknowledging the signs and symptoms of the swallowing problem, at times obtaining further information through a meal observation, and formulating recommendations as indicated and communicating results of the screen to the interdisciplinary care team. A swallow function assessment is performed to evaluate the efficiency of oral preparatory and oral, pharyngeal, and cervical esophageal swallowing, and does not define dysphagia according to airway safety alone (i.e., aspiration). It is important in evidence-based practice that practitioners have a clear understanding if an instrument is to be used as a screening or assessment tool.

Critical appraisal of the current study identified methodological violations regarding the following: patient selection based on features suggestive of dysphagia; failure to select a psychometrically sound screening tool for comparison; failure to use rater blinding; failure to use intra- and inter-rater reliability; and failure to use a parallel design between screening tools. Each of these methodological violations placed the investigation at substantial risk for bias. For example, introducing a screening tool for a patient with a known dysphagia may inflate the diagnostic accuracy of the instrument, presenting a positive trend. The MWST was compared with the CST in this study to detect silent aspiration and laryngopharyngeal sensory dysfunction. In 2003, Tohara et al. [3] questioned if three non-video fluorographic tests could be used in combination versus independently as an effective screening battery in order to guide clinician practice. The investigators reported the water test alone (i.e., MWST) was comparatively insensitive for predicting aspiration; therefore, selecting to use a comparison tool with limited psychometric validation (i.e., the MWST) infringes upon methodological quality. Failure to use blind administration may evoke bias threats to internal validity and limit credibility. Failure to use intra- and inter-rater reliability may also induce bias. The use of intra- and inter-rater reliability enhances the level of agreement among repeated trials performed by a single rater and a group of raters, respectively; therefore, not using this type of reliability measure may potentially increase Type I error. Finally, failure to use equivalent design between screening tools may also cause bias as well as Type I error. All participants received the MWST 3 times. The lowest score was used for comparison against the CST score which was obtained from one trial. Furthermore, arbitrary cutoff scores were used to determine the presence or absence of dysphagia (i.e., 1-3 dysphagia present; 4, 5 dysphagia absent). Tohara et al. used the MWST and CST assessment criteria in their 2003 investigation; however, the purpose of their investigation was not to determine reliability and validity of the rating scale. To date, no investigations were identified to support this measure.

In order to quantify laryngopharyngeal sensory dysfunction, laryngopharyngeal sensory capacity must be determined by elicitation of the laryngeal adductor reflex (LAR), a sensory-motor response. In order to trigger the LAR, laryngopharyngeal sensory discrimination testing must be performed. This has traditionally been done by delivering an air pulse stimuli through the port of a flexible endoscope to the mucosa innervated by the superior laryngeal nerve (i.e., FEESST) [4]. This was not assessed by the investigators in this study. Instead, the authors made a hypothesis based on observations during acidic water swallows that stronger and faster swallowing was elicited in dysphagia patients when sensory input was enhanced by acid stimulation. The authors did describe the pathophysiological mechanism, but this description was theoretical based and not objectively measured.

The investigators recruited 51 participants for this study; however, in order for a new index to be considered reliable it should be rated against a larger sample size. Wilkins et al. [5] reported prevalence rates of dysphagia in an adult unselected primary care population at $22.6 \%$. Using a confidence interval of $95 \%$ and a margin of error of $2.5 \%$ the investigators would have been required to recruit approximately 188 participants for the study described to improve the reliability of the findings.

The value of a test is commonly ascertained by calculating sensitivity and specificity. Kobayashi et al. defined sensitivity of a clinical sign given $3 \mathrm{~mL}$ of $2.0 \% \mathrm{w} / \mathrm{v}$ of a citric acid solution as (1) no swallow, (2) swallow/dyspnea, or (3) swallow/cough or wet-hoarseness for detecting a sign on video-endoscopy (e.g., aspiration) as the proportion of patients who had aspiration on video-endoscopy and who were also positive for one of three clinical signs. The specificity of the clinical sign is defined as the proportion of patients who did not have the sign on video-endoscopy (e.g., aspiration) who also did not have any clinical signs, as in (4) swallow/no dyspnea/no cough no wet- 
Citation: Baylow HE (2016) A Review Article of A Citric-Acid-Solution Swallowing Test as a Screening Test for Aspiration at Bedside and for the Early Detection of Swallowing Dysfunction. Int J Neurorehabilitation 3: 201. doi:10.4172/2376-0281.1000201

Page 2 of 2

hoarseness/no two dry swallows or (5) swallow/no dyspnea/no cough no wet-hoarseness/two dry swallows. Although sensitivity was high (94.4\%) specificity was only moderately-high (69.7\%). For a screening tool to be considered a reliable measure it would need to generate both high sensitivity and specificity in order to either "rule in" or "rule out" the clinical sign for detecting aspiration. The authors went on to report sensitivity and specificity measures for swallowing dysfunction. Although these values are promising, it is unclear what is being measured that is different for aspiration.

To safeguard patient care, research design and methodological accuracy must be at the forefront of evidence-based practice. The idea of a citric-acid-solution swallowing test may be beneficial in providing information regarding the pattern generating neural circuitry of swallowing; however, further research and extensive reliability testing is warranted prior to its implementation into the clinical arena. Concurrent validity could be established against a screen such as The Toronto Bedside Swallowing Screening Test (TOR-BSST) [6].

\section{References}

1. Kobayashi K, Kobayashi M, Abo M (2015) A citric-acid-solution swallowing test is useful as a screening test for aspiration at bedside and for the early detection of swallowing dysfunction. J Clin Trials 5: 1-4.

2. (2004) American Speech-Language-Hearing Association. Preferred practice patterns for the profession of speech-language pathology.

3. Tohara H, Saitoh E, Mays KA, Kuhlemeier K, Palmer JB (2003) Three tests for predicting aspiration without video fluorography. Dysphagia 18 : 126-134.

4. Aviv JE (2000) Prospective, randomized outcome study of endoscopy versus modified barium swallow in patients with dysphagia. See comment in PubMed Commons below Laryngoscope 110: 563-574.

5. Wilkins T, Gillies RA, Thomas AM, Wagner PJ (2007) The prevalence of dysphagia in primary care patients: a HamesNet Research Network study. J Am Board Fam Med 20: 144-150.

6. Martino R, Silver F, Teasell R, Bayley M, Nicholson G, et al. (2009) The Toronto Bedside Swallowing Screening Test (TOR-BSST): development and validation of a dysphagia screening tool for patients with stroke. Stroke 40: 555-561. 